\section{Beatmungspatienten: orale bzw. enterale Dekontamination und Blutstrominfektionen}

Wittekamp B et al. Decontamination strategies and bloodstream infections with antibiotic resistant microorganisms in ventilated patients: A randomized clinical trial. JAMA 2018; 320: $2087-2098$

Die Frage, ob eine orale Dekontamination mit antiseptischen Substanzen oder Antibiotika bzw. eine selektive Darmdekontamination bei Beatmungspatienten einen Vorteil bringt, wird nach wie vor kontrovers diskutiert.

Als möglicher Confounder und Erklärung für widersprüchliche Studiendaten wird hierbei die epidemiologische Ausgangslage hinsichtlich der Resistenzlage der für Infektionen verantwortlichen Erreger genannt.

Wittekamp und Mitarbeiter verglichen daher die Mundspülung mit Chlorhexidin (CHX) mit einem selektiven oralen Dekontaminationsregime (SOD), bestehend aus Mundpaste mit den Wirkstoffen Colistin, Tobramycin und Nystatin, einem selektiven Darmdekolonisationsregime (SDD), bestehend aus Mundpaste und gastrointestinaler Suspension, bestehend aus den gleichen Antibiotika und einer Standardgruppe (Baseline), in der alle Patienten täglich äußerlich mit Chlorhexidin $2 \%$ gewaschen wurden und ein Händehygieneprogramm implementiert wurde. An der Studie beteiligten sich zwischen Dezember 2013 und Mai 2017 13 europäische Intensivstationen, auf denen Blutstrominfektionen in mehr als $5 \%$ der Fälle durch Extended-Spectrum- $\beta$ Laktamasebildende (ESBL) Enterobacteriaceae verursacht wurden.

Als primärer Outcomeparameter wurde die Rate von auf der Intensivstation erworbenen Blutstrominfektionen mit multiresistenten gramnegativen Erregern (MRGN) gemessen, als sekundärer Outcomeparameter die 28-Tage-Mortalität. Eingeschlossen wurden Patienten mit mehr als 24-stündiger zu erwartender Be- atmungsdauer. Insgesamt konnten 8665 Patienten in die Studie eingeschlossen werden (2251 Baseline, 2108 CHX, 2224 SOD, 2082 SDD). MRGN-Blutstrominfektionen traten bei 144 Patienten mit 154 Episoden auf. Der Anteil in den Studiengruppen betrug 2,1\% (Baseline), 1,8\% (CHX), 1,5\% (SOD), 1,2\% (SDD), wobei die adjustierten Risikoraten gegenüber der Baseline 1,13 (95\%-Konfidenzintervall $0,68-1,88$ ) in der CHX-Gruppe, 0,89 (95\%-Konfidenzintervall $0,55-1,45$ ) in der SOD-Gruppe und 0,7 (95\%-Konfidenzintervall $0,43-1,14$ ) betrugen.

Auch die Mortalitätsunterschiede waren mit adjustierten Odds Ratios von 1,07 (95\%-Konfidenzintervall $0,86-1,32$ ) in der CHX-Gruppe, 1,05 (95\%-Konfidenzintervall $0,85-1,29$ ) in der SOD-Gruppe und 1,03 (95\%-Konfidenzintervall 0,81,32) gegenüber der Baseline-Gruppe nicht statistisch signifikant unterschiedlich.

FAZIT

Die Autoren kommen zu dem Schluss, dass Dekolonisationsregimes mit oralen Antiseptika, oralen Antibiotika oder oralen und enteralen Antibiotika keine Vorteile hinsichtlich der Entwicklung von MRGN-Blutstrominfektionen oder der Mortalität bei beatmeten Patienten in einem Setting mit moderater bis hoher endemischer Resistenzbelastung erbringen. Die Autoren diskutieren einige Limitationen ihrer Studie, insbesondere die gewählte Cluster-Randomisierung nach der Baseline-Phase, die lediglich einen Post-hoc-Vergleich zwischen den unterschiedlichen Regimes ermöglicht, sowie die nicht erreichte initial berechnete Power von 10800 Patienten, wobei die Post-hoc-Power-Analyse lediglich für die Blutstrominfektionen eine Möglichkeit der Effektivität von SDD in einer größeren Studie nicht ausschließen kann, jedoch hinsichtlich der Mortalität auch in einer größeren Studienpopulation kein anderes Ergebnis zu erwarten wäre.

PD Dr. Sebastian Schulz-Stübner, Freiburg

\section{Kommentar}

Waren bisherige Studien zu antiseptischen Mundspülungen, SOD oder SDD meist auf das primäre Outcome Pneumonie fokussiert, wählten die Autoren dieser Studie Blutstrominfektionen als primären Outcomeparameter und tragen damit dem vermuteten Mechanismus einer bakteriellen Translokation durch eine gestörte Mukosabarriere Rechnung. Außerdem untersuchten sie die Interventionen im Gegensatz zu vielen niederländischen Arbeiten, die in einem Setting mit niedriger endemischer Resistenzrate stattfanden, in Intensivstationen mit verhältnismäßig hohen ESBL-Raten.

Angesichts des negativen Ergebnisses der Studie bleibt allerdings die Frage offen, ob das gewählte Einschlusskriterium einer zu erwartenden Beatmungsdauer von mehr als 24 Stunden tatsächlich die richtigen Risikopatienten für eine derartige Komplikation und damit gegebenenfalls für eine mikrobiotabeeinflussende Intervention widerspiegelt.

Interessant sind außerdem einige $\mathrm{Ne}$ benaspekte der Studie. So ergaben sich unter den jeweiligen Dekontaminationsregimes keine Verschiebungen der allgemeinen Resistenzlage auf den beteiligten Stationen, obwohl in einem Zentrum die Studie wegen eines klonalen Ausbruchs mit einer Colistin-resistenten Klebsiella pneumoniae und in einem anderen Zentrum wegen eines krankenhausweiten, polyklonalen Ausbruchs mit Carbapenem-resistenten Enterobacteriaceae unterbrochen werden musste.

In der Chlorhexidin-Gruppe wurde CHX $2 \%$ imVerlauf durch $\mathrm{CHX} 1 \%$ ersetzt, da in den 2 beginnenden Zentren bei 9,8\% der mit $\mathrm{CHX} 2 \%$ behandelten Patienten lokale Komplikationen im Sinne von oropharyngealen Schleimhautschäden auftraten. Der Gesamtantibiotikaverbrauch änderte sich über die Zeit ebenfalls nicht.

Die Autoren diskutieren, ob das Fehlen der systemischen Gabe eines Drittgenerations-Cephalosporins, die sonst klassischer Bestandteil von SDD-Regimes war, in ihrer SDD-Gruppe Auswirkungen ge- 
habt haben könnte und berechneten daher, wie viele Patienten innerhalb der ersten 4 Tage eine Blutstrominfektion mit einem Drittgenerations-Cephalosporin-empfindlichen Erreger erlitten hatten. Auch unter Ausschluss dieser Patienten hätten sich jedoch keine signifikanten Ergebnisse oder Verbesserungen der Mortalität wie in älteren niederländischen Studien [1] gezeigt.

Trotz des pathophysiologisch plausiblen Mechanismus der oralen und enteralen Darmdekontaminationsregimes (die man vielleicht besser als darmmikrobiotabeeinflussende Prophylaxe [MBP] bezeichnen sollte) bei nun wiederholt nachgewiesener relativer Sicherheit hinsichtlich der Resistenzentwicklung bleibt die Suche nach den richtigen Indikationen und anstelle einer universellen Anwendung sind individualmedizinische Konzepte gefragt.

PD Dr. Sebastian Schulz-Stübner,

Freiburg

Literatur

[1] de Smet AM, Kluytmans JA, Cooper BS et al. Decontamination of the digestive tract and oropharynx in ICU patients. N Engl J Med 2009; 360: $20-31$ 